\title{
Generation of Quantum Vector States Using the Exchange Interaction
}

\author{
Mrittunjoy Guha Majumdar \\ University of Cambridge, Cambridge, UK \\ Email: mgm913@gmail.com
}

How to cite this paper: Majumdar, M.G. (2019) Generation of Quantum Vector States Using the Exchange Interaction. Journal of Applied Mathematics and Physics, 7, 3116-3131.

https://doi.org/10.4236/jamp.2019.712219

Received: November 8, 2019

Accepted: December 16, 2019

Published: December 19, 2019

Copyright $\odot 2019$ by author(s) and Scientific Research Publishing Inc. This work is licensed under the Creative Commons Attribution International License (CC BY 4.0).

http://creativecommons.org/licenses/by/4.0/

\section{c) (i) Open Access}

\begin{abstract}
In this paper, I study the invariant subspaces of quantum states under $S W A P^{a}$ gates arising from the exchange interaction and their use in quantum computation. I investigate the generation and characterization of invariant-subspace vector-states that arise from such gates. I also state a condition for the locus of states that are accessible using the $S W A P^{\alpha}$ gates, given an initial input state.
\end{abstract}

\section{Keywords}

Quantum Entanglement, Vector States, Exchange Interaction

\section{Introduction}

The non-local physical phenomena related to quantum entanglement [1] [2] [3] have played a pivotal role in the development of quantum mechanics during the last century. Entanglement in many-particle states has been extensively studied [4] [5] [6], and such states are known to have symmetries that help in determining their entanglement properties [7] [8]. This is useful in determining the usability of these quantum states as resource-states for application in quantum information processing tasks [9]-[14]. Prior to using these resource-states, the generation of these quantum states is important, and this is an arduous task that requires a high degree of control. Quantum state engineering has been a way to generate arbitrary quantum states experimentally [15]-[19]. The existence of entanglement equivalence classes [20] of quantum states, particularly symmetric states, simplifies the experimental generation of these states. By producing a single state of an equivalence class, the entire subspace of states in that class can be accessed using a reduced set of operations. As a result of this, the characterization of symmetries in quantum states goes conjointly with the study of re- 
source-states for quantum information applications, for example, quantum operation sharing [21] and super-dense coding [22] [23].

A form of symmetry that has been of significant interest to the world of quantum information is that of symmetry under permutations [24] [25] [26] [27] [28]. An early example of a success of the systematic mathematical study of permutation-symmetric states was the discovery of noiseless subspaces [25], in which quantum states can evolve without the introduction of bit-flip errors. This follows from the inherent parity constraints on permutation symmetric states. Permutation symmetric states (e.g. Dicke states) can occur as ground states in certain physical systems, some of which have been recently implemented experimentally [29] [30] [31]. A (partial) permutation on two qubits can be carried out by a (partial) SWAP gate [32]. Moreover, the $S W A P^{\alpha}$ gate, together with single-qubit operations, is found to produce a universal set of quantum gates (enabling any quantum computation) [33]. These two-qubit gates can be realised in several physical systems. In electronic systems, the exchange interaction provides a natural framework for the implementation of the $S W A P^{\alpha}$ gate [34] [35], but equivalent implementations also exist for atomic systems [36] [37], nitrogen vacancies [38] and photonic systems [39] [40] [41] [42].

Burkard et al. [43] showed how the $\sqrt{\mathrm{SWAP}}$ gate can be used to create a controlled phase-flip gate (CPHASE), which in turn can be used to create a XOR gate. Barenco et al. [44] showed that any unitary two-qubit gate can be simulated by four one-bit gates and two XOR gates, thereby effectively leading to universal quantum computation using only the $\sqrt{\mathrm{SWAP}}$ and single qubit rotation gates . Since local single-qubit operations are difficult to implement in hardware, $D i$ vincenzo et al. subsequently looked at how universal quantum computation can be achieved by using only $S W A P^{\alpha}$ gates if one encodes pseudo-spin qubits using three physical qubits [33]. This naturally leads to the question of whether universal quantum computation could be implemented purely using $S W A P^{a}$ with a specific value of $\alpha$. It was shown by Tanamoto et al. [45] that upon preparing the initial state as $| \pm\rangle=\frac{1}{2}(|0\rangle+|1\rangle)(|0\rangle-|1\rangle)$ and applying the $\sqrt{\text { SWAP }}$ one can obtain Raussendorf's cluster state [46] but only after two single-qubit rotations.

In this paper, I am interested in the general question of what kinds of states are accessible from an arbitrary quantum state, which is easier to produce in physical systems than entangled states, using the $S W A P^{\alpha}$ operators and what are their applications? Our approach to this is to determine the invariant subspaces of the permutation group $S_{n}$ when applied to the Hilbert space of $n$-qubits. These subspaces comprise vectors that map back onto a linear superposition of the same vectors under the action of $S W A P^{\alpha}$ operators. In Section II, we look more closely at the invariant subspaces for the combinations of $S W A P^{\alpha}$ gates and look at the kinds of invariant subspaces that can arise for quantum states with different numbers of $|0\rangle$ and $|1\rangle$ in them. In Section III, we highlight the numerical complexity of the problem and look closely at the analytical method 
to generate vector states for our quantum system. In Section IV, we look at the idea of symmetry under parity of this system and discuss an efficient algorithm for generating $n$-qubit vector states for $S W A P^{\alpha}$ gates. In Section V, we look at the problem of accessibility of quantum states using Power-of-SWAP gates and devise conditions for the output states, given aninput state and combination of $S W A P^{\alpha}$ gates.

\section{SWAP ${ }^{\alpha}$ and Invariant Subspaces}

Quantum logic gates are essential building blocks of a quantum computer. The $S W A P^{\alpha}$ quantum gate with $0<\alpha \leq 1$ is one of the most efficient quantum gates in two-qubit quantum computation, with three $S W A P^{\alpha}$ gates combined with six single-qubit gates being able to realize any arbitrary two-qubit unitary operation [47] [48] [49]. The $S W A P^{a}$ gate can be experimentally implemented in several physical systems such as in the exchange interaction between electrons trapped by surface acoustic waves [34] [50]. In our paper, we look at the use of only $S W A P^{a}$ operators for generation of quantum states and our analysis applies to any $n$-qubit quantum state that can undergo $S W A P^{\alpha}$ operations, where $\alpha$ is any real number.

We find that a combination of all $S W A P^{\alpha}$ gates between a finite number of qubits comprise a group that is isomorphic to the Symmetric Group $S_{n}$, which is the group of all permutations or self-bijections of a set of nelements with the operation of composition, and that only certain points in the Hilbert Space are accessible using the $S W A P^{\alpha}$ gates, given a particular input state. To find the kinds of states that are accessible under the repeated action of a $S W A P^{\alpha}$ operator, we need to study the invariant subspaces ${ }^{1}$ associated with this combination of $S W A P^{\alpha}$ operators. We find the structure of the invariant subspaces of the combination of $S W A P^{\alpha}$ gates to be the same for all $\alpha$.

The invariant subspaces of a vector space under the action of a group emerge from the irreducible representations of the group. For any group, the number of irreducible representations is equal to the number of conjugacy classes $^{2}$. It is seen that for the Symmetric Group $S_{n}$, the way in which we can permute $n$ elements without changing the structure of the superposition of vector states characterize the specific conjugacy class, and by extension the invariant subspace of the group, associated with those vector states. In other words, the invariant subspaces of the Symmetric Group $S_{n}$ comprise of vector states, whose linear superposition remains invariant under the action of permutation of elements within the partition that corresponds to the invariant subspace. For instance, in the case of permutation symmetries of four elements, we have conjugacy classes of the Symmetric Group $S_{4}$ such as [4] (cyclic permutation of all four ele-

\footnotetext{
${ }^{1} \mathrm{~A}$ subspace $W$ of a vector space $V$ is invariant, with respect to a group $G$, if $\rho(g) W \subset W$ for every $g \in G$, where $\rho$ is a representation of the group $G$ of the group $S_{n}$.

${ }^{2} \mathrm{~A}$ Conjugacy Class is an equivalence class that, for every element $a$ in a group $G$, contains $b \in G$ such that there exists $g \in G$ with $b=g_{a g}{ }^{-1}$.
} 
ments), [31] (transposing three, leaving one alone) and [1111] (no change) ${ }^{3}$. A point to note here is that there could be several cases for each cycle pattern. For instance, for four qubits 1-2-3-4, (12) (34) and (13) (24) are specific [22] cycles that correspond to a $T_{12} T_{34}$-invariant and $T_{13} T_{24}$-invariant subspaces respectively, where $T_{i j}$ correspond to transposition of the $i^{\text {th }}$ and $f^{\text {th }}$ qubit.

All the vector states that are associated with any specific invariant subspace corresponding to the Symmetric Group $S_{n}$ are found to have the same Hamming weight in their qubit representation. We find that the number of basis vectors comprising a specific invariant subspace and the forms of these vector states are related to the kind of permutation permissible within the partition corresponding to the invariant subspace. For $n$-qubit states, those vector states that span the entire subspace of states with a particular Hamming weight in as close to an equiprobabilistic manner (over the basis vectors) as possible are found to be closer to being invariant under all possible permutations of the $n$ qubits, and are found to be part of one-dimensional invariant subspaces (with only one constituent vector state). For instance, the three-qubit quantum state that is invariant under all permutation under $S_{3}$ of its qubits is $|\psi\rangle=\frac{1}{\sqrt{3}}(|001\rangle+|010\rangle+|100\rangle)$ and this is the only vector comprising the invariant subspace corresponding to the trivial irreducible representation for three-qubit states with Hamming weight 1 in their qubit representation.

The number of $|0\rangle$ and $|1\rangle$ qubits in multiqubit states, represented by their Hamming weight, is found to uniquely define the kinds of invariant subspaces, with their associated permutation symmetries and corresponding partitions, of the $S W A P^{\alpha}$ gates we can create with those states.

Let us consider a quantum state of the form

$$
|\psi\rangle=\sum_{\operatorname{PERM}(i)} \alpha_{i}|\underbrace{00 \cdots 0}_{k} \underbrace{11 \cdots 1}_{n-k}\rangle_{i}
$$

where $i$ is an index related to each permutation of the qubits and the summation is over $i$, all the permutations of the qubits. We find that only certain permutations (and ways of partitioning) are applicable and important for a given set of vector-states that have a specific value of $k$. The change in an arbitrary superposition of the vector states resulting from these specific permutation operations can be used to express the change in the superposition of these vector states under any other permutation operation. In this respect, for $n$-qubit states with even $n$ and $k \leq \frac{n}{2}$, we find that the relevant partitions (and corresponding permutation symmetries) are $\mathcal{C}^{(k)}=\left\{C_{i}^{(k)}\right\}=\{[n],[n-1,1], \cdots,[n-k, k]\}$, where the $i$ is the index of the partitions for a fixed value of $\$ \mathrm{k} \$$. This is because the symmetries under permutations $[n-(k+1), k+1], \cdots,[11 \cdots(n$ times $)]$ are encapsu${ }^{3}$ Here, we use the notation $\left[t_{1}, t_{2}, \cdots\right]$ to represent a transposition where $t_{1}$ qubits are rotated by one among themselves, $t_{2}$ qubits by one among themselves, and so on. 
lated in the existing set $C_{i}$. An example of this is shown in Figure 1 for $k=2$, where the permutation within the partition $[n-3,3]$ is shown to be equivalent to permutation within the partition $[n]$. For $n$ qubits states with even $n$ and $k>\frac{n}{2}$, the relevant partitions are $C_{i}^{(k)}=\{[n],[n-1,1], \cdots,[n-(n-k), n-k]\}$. If we consider all possible values of $k$ for a fixed value of $n$, the total number of sets of vector-states that correspond to the partition $[n]$ is $(n+1)$. Similarly, we have $(n-1)$ sets of basis-vectors for the partition $[n-1,1],(n-3)$ for the partition $[n-2,2]$ and so on till we have 1 set for the partition $\left[\frac{n}{2}, \frac{n}{2}\right]$, with there being $\frac{n}{2}+1$ kinds of subspaces that are relevant. As a result, $N_{\text {inven } n)}^{(\text {eve }}=\frac{1}{2}\left(\frac{n}{2}+1\right)((n+1)+1)=\frac{1}{4}(n+2)^{2}$. For odd $n$, we have conjugacy classes $C_{i}^{(k)}=\{[n],[n-1,1], \cdots,[n-k, k]\}$ for $k \leq \frac{n-1}{2}$ and $C_{i}^{(k)}=\{[n],[n-1,1], \cdots,[n-(n-k), n-k]\}$ for $k \geq \frac{n+1}{2}$. We have $(n+1)$ basis vector-sets associated with the invariant subspace $[n],(n-1)$ for $[n-1,1],(n-3)$ for $[n-2,2]$ and so on till 2 sets for $\left[\frac{n+1}{2}, \frac{n-1}{2}\right]$, with there being $\frac{n-1}{2}+1$ kinds of partitions that need be independently described and are relevant. As a result, $N_{i n v}^{(\text {odd } n)}=\frac{1}{2}\left(\frac{n-1}{2}+1\right)((n+1)+2)=\frac{1}{4}(n+1)(n+3)$. Thus, we find that the number of invariant subspaces, $N_{i n v}$, for an $n$-qubit system, operated upon by the $S W A P^{\alpha}$, is given by:

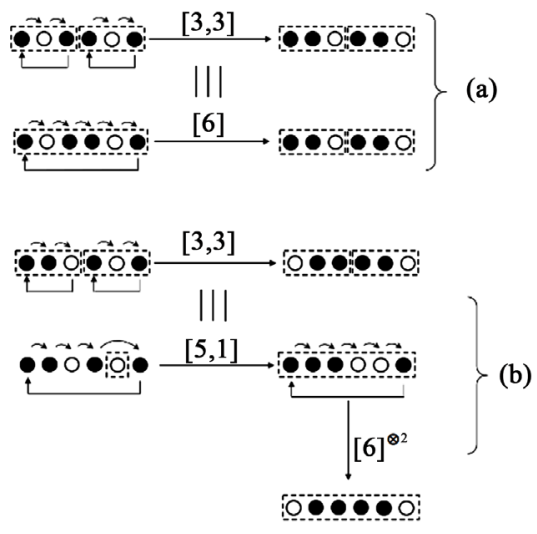

Figure 1. Permutations for $n=6, k=2$ state: In this case, we have four qubits of one kind (say $|1\rangle$ ) and 2 qubits of the other kind (say $|0\rangle$ ). (a) the $|0\rangle$ qubits have two $|1\rangle$ qubits in between; rotating the first three qubits among themselves, while rotating the next three qubits among themselves, is equivalent to rotating all the qubits (to the right) once; (b) the $|0\rangle$ qubits have one $|1\rangle$ qubit in between, as shown; then carrying out the same operation: rotating the first three qubits among themselves, while rotating the next three qubits among themselves, is equivalent to rotating by a $[5,1]$ transposition followed 
by two instances of the complete rotation [6].

$$
N_{i n v}=\left\{\begin{array}{l}
\frac{1}{4}(n+2)^{2}, \text { if } n \text { is even } \\
\frac{1}{4}(n+1)(n+3), \text { if } n \text { is odd }
\end{array}, n>1 .\right.
$$

Invariant subspaces of the Symmetric Group $S_{n}$ and reducibility of representations are closely linked [51]. If a representation $\rho$ of the group $G$ has no non-trivial invariant subspace, we say that it is irreducible. Defining $U_{\{\operatorname{SWAP}\}}\left(\left|q_{\{i\}}\right\rangle,\left|q_{\{j\}}\right\rangle\right)$ as the SWAP operation between the $i_{t h}$ and $j_{t h}$ qubits in an n-qubit state $\left|q_{1} q_{2} \cdots q_{\{i\}} \cdots q_{\{j\}} \cdots q_{\{n\}}\right\rangle$, we find that the matrix representation $U_{\{\mathrm{SWAP}\}}\left(\left|q_{\{i\}}\right\rangle,\left|q_{\{j\}}\right\rangle\right)$ can be transformed into a block form $D$, comprising of irreducible representations of the Symmetric Group $S_{n}$ associated with each invariant subspace, with a similarity (equivalence) transformation $S$. If one has $r$ distinct SWAP operations between qubits applied consecutively, then the resultant operation can be block-diagonalized as follows:

$$
D=D_{1} D_{2} \cdots D_{r}=\left(S^{-1} U_{\text {SWAP }}^{1} S\right)\left(S^{-1} U_{\text {SWAP }}^{2} S\right) \cdots\left(S^{-1} U_{\text {SWAP }}^{r} S\right)
$$

where $U_{\text {SWAP }}^{i}$ represent the $i^{\text {th }}$ distinct SWAP operation.

This similarity transformation is found to be useful in block-diagonalizing the basic $S W A P^{\alpha}$-gates representations as well, since by definition,

$$
U_{\mathrm{SWAP}^{\alpha}}=\frac{1-\mathrm{e}^{i \pi \alpha}}{2} U_{\mathrm{SWAP}}+\frac{1+\mathrm{e}^{i \pi \alpha}}{2} I_{(2 \times 2)}
$$

where $I_{(2 \times 2)}$ is a $2 \times 2$ identity matrix. As a result, the similarity transformation $S$ that transforms the composite SWAP operator to a block-diagonal form: $D=S^{-1} U_{\text {SWAP }} S$, will also transform the $U_{\text {SWAP }^{\alpha}}$ in the same block form, up to certain additive terms: $S^{-1} U_{\text {SWAP }^{\alpha}} S=\frac{1-\mathrm{e}^{i \pi \alpha}}{2} D_{1}+\frac{1+\mathrm{e}^{i \pi \alpha}}{2} I_{(2 \times 2)} \equiv D^{\prime}$. Due to the relation established in (2), the similarity transformation and associated invariant subspace associated with $U_{\text {SWAP }^{\alpha}}$ must be of the same structural form as those for the $U_{\text {SWAP }}$ gate. This is also true for a combination of $U_{\text {SWAP }^{\alpha}}$ gates: $\left(S^{\otimes n}\right)^{-1}\left(U_{\text {SWAP }^{\alpha}}^{\otimes n}\right)\left(S^{\otimes n}\right)=\left(S^{-1} U_{\operatorname{SWAP}^{\alpha}} S\right)^{\otimes n}$.

The matrix decomposition thus formed is block diagonal, as shown in the illustrative example in Figure 2 for the case of two matrices. Thus, we find that the various combinations of $n$ copies of $S W A P^{\alpha}$ gates, operating over a multi-qubit input state, produce a group that is isomorphic to the symmetric group $S_{n}$. In fact, any function of $S W A P^{\alpha}$ gates can be put into the block form using the same similarity transformation: $S^{-1} f\left(U_{\mathrm{SWAP}^{\alpha}}\right) S=f(0) S^{-1} I_{2 \times 2} S+$ $f^{\prime}(0) S^{-1} U_{\text {SWAP }^{\alpha}} S+f^{\prime \prime}(0) \frac{1}{2 !} S^{-1} U_{\text {SWAP }^{\alpha}} S S^{-1} U_{S W A P^{\alpha}} S+\cdots$, using Taylor series expansion of the function of $U_{\text {SWAP }^{\alpha}}$, and pre- and post-multiplying with $S$ and $S^{-1}$ respectively. As a result, the determination of the similarity transformation for SWAP alone gives us a useful tool for finding the invariant subspaces and associated vectors for any arbitrary combination or function of partial SWAP 
gates.
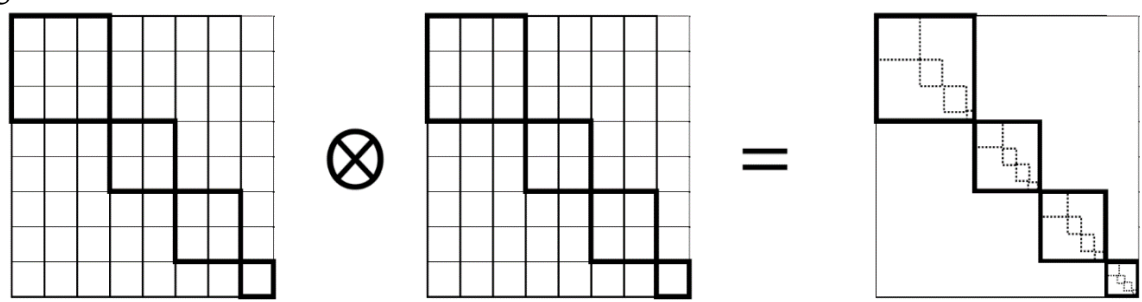

Figure 2. Illustrative example of tensor Product of two block diagonal matrices with one $3 \times 3$ block, two $2 \times 2$ blocks and one single block. For the case of block-diagonal matrices $D$, the combination of such matrices leads to a resultant block-diagonal matrix, thereby showing that various combinations of $n$ copies of $S W A P^{\alpha}$ gates, operating over a multi-qubit input state, produce a group that is isomorphic to the symmetric group $S_{n}$.

\section{Generation of Vector States}

Due to the non-commutativity of adjacent $S W A P^{a}$ gates with at least one common qubit, the number of output states generated for a given input state and gate-combination is a lot more than in the case of commutative gates. For an -qubit separable input state and $k$ gates used, we have the following number of cases and associated accessible output states:

$$
N_{a c c}^{l}=l^{k} \times 2^{l} .
$$

As can be seen, the problem grows exponentially with the number of qubits in the input (separable) states. The number of gates also scales this value exponentially. Certain cases are degenerate but the problem, all in all, grows exponentially with the number of qubits in the input state and the number of gates being operated with on the input state. As a result, this is a problem that cannot be treated efficiently using numerical methods, as the number of qubits involved increase. To resolve this problem, we look at analytic methods, using techniques for the representation theory for the symmetric group $S_{n}$.

The system we are considering is an arbitrary $n$-qubit state and we are operating combinations of $S W A P^{\alpha}$ operations on such an input state. The action of the is: $|00\rangle \rightarrow|00\rangle, \quad|01\rangle \rightarrow \frac{1+\mathrm{e}^{\{i \pi \alpha\}}}{2}|01\rangle+\frac{1-\mathrm{e}^{\{i \pi \alpha\}}}{2}|10\rangle$, $|10\rangle \rightarrow \frac{1+\mathrm{e}^{\{i \pi \alpha\}}}{2}|10\rangle+\frac{1-\mathrm{e}^{\{i \pi \alpha\}}}{2}|01\rangle,|11\rangle \rightarrow|11\rangle$. The relevance of the SWAP or partial SWAP arises from the fact that any permutation of elements can be expressed as a combination of transpositions [52], which on the physical level can be realized using SWAP operations. The matrix representation for these SWAP operations can be found efficiently using a standard sorting algorithm operated on a $2^{n} \times 2^{n}$ identity matrix and permuting the relevant rows of the matrix depending on which qubits are being swapped by the particular SWAP operation. This is efficient since instead of finding all the $n$ ! permutation matrices using a scheme such as that based on Cayley trees, one can simply use $n^{2}-n$ transpositions to generate the group. Any arbitrary element of the group can then be 
found using a combination of these transpositions.

The next significant step in the process of generating the vector states is to look at the irreducible representations $\left\{D_{i}, i=1,2, \cdots, N !\right\}$ of the group. The first step in this regard is to find the dimensions of the irreps using the Young's Tableau [53]. For any $n$ qubit system, we can find the dimensionality of the irrep using the hook lengths of the elements in it [54] [55] and the hook product [56] $h$ of the tableau. The dimension $d$ of the irrep is then given by

$$
d=\frac{n !}{h} .
$$

Once we have found the dimensions of the irreps, the next step is to find the forms of the irreps. We find the Young's orthogonal form for the irreps using the equation [57]

$$
\begin{aligned}
& D^{[\mu]}((k, k+1))\left\{e_{M_{1} \cdots M_{n}}^{[\mu]},\right. \\
& =\left(\rho\left(M_{1} \cdots M_{n} ; k+1, k\right)\right)^{\{-1\}} \hat{e}_{M_{1} \cdots M_{n}}^{[\mu]}+A \hat{e}_{M_{1} \cdots M_{k+1} M_{k} \cdots M_{n}}^{[\mu]},
\end{aligned}
$$

where $A=\sqrt{1-\left(\rho\left(M_{1} \cdots M_{n} ; k+1, k\right)\right)^{-2}}$ and where we use the orthonormal basis $\hat{e}_{\{i\}}^{\mu}$ where $i=1,2,3, \cdots, f[\mu]:\left(\hat{e}_{i}^{[\mu]}, \hat{e}_{j}^{[\mu]}\right)=\delta_{i j}$. The index $i$ of the basis-vector is related to the Yamanouchi symbol $M$ for the tableau [53].

Using this formula, we can look at the irreducible representations for all elements of the group. A point to note here is that not all irreducible representations may be required to describe a physical system. A good way to see which irreducible representations are relevant, we must construct a Character Table. We do so by considering the trace (character) values of the irreps for a given conjugacy class and kind of irrep (as defined by its dimensionality). The columns of the Character Table are for elements of the same conjugacy class while the rows of the Table are for elements of the same irrep. Once we have constructed the entire Character Table, we assign a variable (say $\alpha_{i}$ for the $i^{\text {th }}$ irrep) to each irreducible representation, and multiply these with the character of the irrep (say $a_{i}$ for the $i^{\text {th }}$ irrep) for each conjugacy class, before adding them up. We equate this sum to the trace (character) of the permutation matrix for each conjugacy class $\xi$ that we found previously:

$$
\xi=\sum_{i} \alpha_{i} a_{i} .
$$

This leads to a system of linear equations, whose solutions determine uniquely the kinds of irreps that are relevant along with the number of irreps of each kind that constitute the block-diagonalized form of the permutation matrices. The permutation matrices $\left\{P_{i}, i=1,2, \cdots, N\right.$ ! $\}$ can be simultaneously diagonalized to irreps $\left\{D_{i}, i=1,2, \cdots, N !\right\}$ with the same block form using a similarity transformation. These two sets of matrices are related by the relation:

$$
D_{i}=S^{\{-1\}} P_{i} S, i=1,2,3, \cdots, N \text { ! }
$$

Solving this set of linear equations algebraically, gives us a similarity transformation $S$. However, this solution is not unique and there may be several un- 
resolved elements within the matrix representation of the similarity transformation $S$. For our system, we resolve this problem using the imposition of a condition of unitarity on this matrix.

Let us say we had a permutation operator $P$ operating on an initial state $|\psi\rangle$ to give a final output state $\left|\psi^{\prime}\right\rangle$ :

$$
\left|\psi^{\prime}\right\rangle=P|\psi\rangle \text {. }
$$

Then operating with the similarity transformation $S^{-1}$, gives us:

$$
S^{-1}\left|\psi^{\prime}\right\rangle=S^{-1} P|\psi\rangle=\left(S^{-1} P S\right) S^{-1}|\psi\rangle=D S^{-1}|\psi\rangle .
$$

Therefore, the eigenvectors of the block-diagonalized matrices are of the form:

$$
|\phi\rangle=S^{-1}|\psi\rangle \text {. }
$$

Usually $|\psi\rangle$ is taken to be the basis comprising of all $2^{n}$ separable $n$-qubit states, and the vector $|\phi\rangle$ is regarded as the invariant subspace vectors, since the block-diagonalized forms have blocks corresponding to the invariant subspaces of the system. So, if one was to begin with any linear combination of $n$ qubit vectors that are spanned by the constituent-vectors of an invariant subspace, the state that emerges out of the application of an arbitrary combination of $S W A P^{\alpha}$ gates is always a linear combination of those constituent-vectors of the invariant subspace as well. We find that for $n$-qubits, we have the following number of such vectors

$$
N_{\{v\}}=\left\{\begin{array}{l}
\sum_{i=0}^{\frac{N}{2}}(n+1-2 i) \times d_{[n-i, i]} \text { if } n \text { is even } \\
\sum_{i=0}^{\frac{N-1}{2}}(n+1-2 i) \times d_{[n-i, i]} \text { if } n \text { is odd }
\end{array} .\right.
$$

The vectors that comprise the invariant subspaces have characteristic entanglement patterns. Invariance of the quantum states under Stochastic Local Operations and Classical Communications (SLOCC) is a way to classify these states into entanglement-families [58] [59] [60] [61]. One of the key aspects of this is that since the $S W A P^{\alpha}$ gate commutes with the total $z$-spin operator $S_{\{z\}}=\sum_{v \in V} \sigma_{v}^{z}$, the action of the Power-of-SWAP gate $U_{\text {SWAP }^{\alpha}}$ breaks the Hilbert Space into a direct sum $H \cong \oplus_{k=0}^{N} \Gamma^{k} \quad[6]$, where $\Gamma^{0}=\{|00 \cdots 0\rangle\}$, $\Gamma^{1}=\{|10 \cdots 0\rangle,|01 \cdots 0\rangle, \cdots,|00 \cdots 1\rangle\}, \Gamma^{2}=\backslash\{|11 \cdots 0\rangle,|101 \cdots 0\rangle, \cdots,|00 \cdots 11\rangle\}$ and so on, with $\Gamma^{N}=\{|11 \cdots 1\rangle\}$. These subspaces are found to have distinct symmetries, depending on the number of $|0\rangle$ and $|1\rangle$ qubits in the state. The states for one-dimensional irreps: the perfectly permutation symmetric Dicke states have been studied for the categorization under SLOCC (Stochastic Local Operation and Classical Communication) invariant families using geometric measures of entanglement [10] [62]. In this paper, we look at the relation of entanglement to symmetries (under transposition cycles) in other kinds of irreps relevant for a general $n$-qubit state.

A general $n$-qubit state with the same Hamming weight across all its superposition states can therefore be written as 


$$
|\psi\rangle=\sum_{i}^{d} a_{i}|i\rangle
$$

where $|i\rangle$ are the vectors associated to the invariant subspace with that Hamming weight and $d$ denotes the dimensionality of the invariant subspace under consideration. More generally, we can express a general quantum state as

$$
|\psi\rangle=\sum_{k}^{l} \sum_{i}^{d_{k}} a_{i}^{(k)}\left|i^{(k)}\right\rangle
$$

where $\left|i^{(k)}\right\rangle$ are the vectors associated to the invariant subspace with the Hamming weight $k$ and $d_{k}$ denotes the dimensionality of the invariant subspace with that particular Hamming weight, while $I$ denotes the number of Hamming weights present in the superposition (system) in the quantum state under consideration.

For four-qubits states, there are 16 vector-states comprising three [31] invariant subspaces each comprising of three vectors, one [22] invariant subspace comprising of two vectors and five trivial representation vector-states. The one-dimensional invariant subspace vectors are the famous Dickestates. These states can be classified into the nine SLOCC-invariant families of entanglement classes defined by Verstaete et al. [58]. Any arbitrary four-qubit state can be written in terms of these invariant subspace vectors, with $l=5$ and

$$
d_{0}=1 ; d_{1}=1,3 ; d_{2}=1,2,3 ; d_{3}=1,3 ; d_{4}=1
$$

in equation (15). This is because there are multiple invariant subspaces for the same Hamming weight, with the Hamming weights 1, 2 and 3 having multiple invariant subspaces in this case. The vectors in an invariant subspace remain within that subspace given any combination of $S W A P^{\alpha}$ gates operated upon them.

\section{Symmetry under Parity and Efficient Algorithm for Generating $n$-Qubit Vector States for SWAP $\alpha$ Gates}

One of the fundamental properties of the $S W A P^{\alpha}$ gates is that they conserve parity. The number of $|0\rangle \mathrm{s}$ and $|1\rangle \mathrm{s}$ in a multiqubit state remain the same. This is a key to proposing an efficient algorithm for finding the vector states associated with the invariant subspaces for the $S W A P^{\alpha}$ gates.

Let us say we have the following families of $\mathrm{n}$-qubit states:

$$
W_{i}=\{\operatorname{PERM}(|\underbrace{\{00 \cdots 0\}}_{\{n-i\}} \underbrace{\{11 \cdots 1\}}_{\{i\}}\rangle)\}
$$

where PERM defines the permutation function for a given set of qubits.

There is no map, $f\left(U_{\mathrm{SWAP}^{\alpha}}\right)$ comprising of only $S W A P^{\alpha}$ that can take us from one such family $W_{i}$ to another family $W_{j}$ for $i \neq j$. This property can be used to define the set of basis vector-sets by starting with a completely symmetric state using all the vectors in the family $W_{i}$ and then finding the null-space vectors for the same. We have found an algorithm and realization of the same that can carry out quick and efficient generation of the vector-states for 
$n$-qubits under operation by $S W A P^{\alpha}$ gates.

\section{Accessibility of Quantum States Using Power-of-SWAP Gates}

In this section, we will be looking at the accessibility of quantum states using only Power-of-SWAP gates for an arbitrary input quantum states. For the two-qubit case, let us begin with a state of the form

$$
\left|\psi_{\{i n\}}\right\rangle=\alpha|00\rangle+\beta|01\rangle+\gamma|10\rangle+\delta|11\rangle .
$$

If we have a parameter $t \in[0,1]$ and the SWAP-gate is the unitary generated by the Heisenberg Hamiltonian for evolution time $t=1$, then we can generate the fractional SWAP by doing the Hamiltonian evolution for time $t \in(0,1)$. For time steps of the form $t=\frac{1}{m}, m \in \mathbb{Z}$, we obtain the so-called $n^{\text {th }}$ Power-of-SWAP $\left(S W A P^{a}\right)$ gates.

In the two-qubit case, let's fix a Power-of-SWAP circuit of depth $m$, such that for each $i=1, \cdots, m$, we apply a $U_{i}=\operatorname{SWAP}^{\gamma_{i}}$ with $\gamma_{i}=1 / n_{i}$ for $n_{i} \in \mathbb{Z}$. Let us look at only the subspace spanned by $\{|01\rangle,|10\rangle\}$. We have

$$
\left|\psi_{\text {in }}\right\rangle \stackrel{U_{m} U_{\{m-1\}} \cdots U_{1}}{\longrightarrow} \psi_{f}=\alpha^{\prime}|00\rangle+\beta^{\prime}|01\rangle+\gamma^{\prime}|10\rangle+\delta^{\prime}|11\rangle,
$$

Since our unitaries are parity preserving, $\alpha^{\prime}=\alpha, \delta^{\prime}=\delta$. Now we can consider the inverse problem: fix the initial and final states, and a precision parameter $\epsilon \in(0,1)$, and compute a sequence $n_{1}, \cdots, n_{m}$ such that $U(\Gamma):=U_{m} U_{m-1} \cdots U_{1}$ brings $\left|\psi_{\text {in }}\right\rangle$ within the $\epsilon$-ball around $\left|\psi_{f}\right\rangle$, i.e.,

$$
\left.|| \psi_{f}\right\rangle-U\left|\psi_{\text {in }}\right\rangle \mid \leq \epsilon \text {. }
$$

Noting that $U(\Gamma)=\mathrm{e}^{i \Gamma H_{\mathrm{SWAP}}}$, we can also first solve for the time parameter $\Gamma$ and then obtain the sequence $\{n\}_{i}$. Let us do this for $\epsilon=0$. Taking the $|00\rangle,|11\rangle$ subspaces as invariant, we have the matrix equation

$$
\frac{1}{2}\left[\begin{array}{ll}
1+\mathrm{e}^{i \pi \Gamma} & 1-\mathrm{e}^{i \pi \Gamma} \\
1-\mathrm{e}^{i \pi \Gamma} & 1+\mathrm{e}^{i \pi \Gamma}
\end{array}\right]\left(\begin{array}{l}
\beta \\
\gamma
\end{array}\right)=\left(\begin{array}{l}
\beta^{\prime} \\
\gamma^{\prime}
\end{array}\right)
$$

which we can solve for $\Gamma$ to get the conditions (assuming \$\alpha\neq \beta $\$$ )

$$
\mathrm{e}^{i \pi \Gamma}=\frac{2 \beta^{\prime}-(\beta+\gamma)}{\beta-\gamma}=-\frac{2 \gamma^{\prime}-(\beta+\gamma)}{\beta-\gamma},
$$

for which to have a solution, we require $\beta^{\prime}+\gamma^{\prime}=\beta+\gamma$.

The result given above can be generalized to the case of higher-number of qubits. For any combination of Power-of-SWAP and general $n$-qubit input state of the form $|\psi\rangle=\alpha_{0}|000 \cdots 00\rangle+\alpha_{1}|000 \cdots 01\rangle+\cdots+\alpha_{2^{N}}|111 \cdots 11\rangle$ being transformed to $\left|\psi^{\prime}\right\rangle=\beta_{0}|000 \cdots 00\rangle+\beta_{1}|000 \cdots 01\rangle+\cdots+\beta_{2^{N}}|111 \cdots 11\rangle$. We derive a condition for the coefficients of the vector basis states with the same Hamming weights. Let denote the set of all coefficients associated with vector states with the same Hamming weight $i$ as $\left\{\alpha_{(h w)^{(i)}}^{k}\right\}$ in the input quantum state and denote the set of all coefficients associated with vector states with the same Hamming 
weight $\left\{\begin{array}{l}\beta_{(h w)}^{k^{\prime}} \\ (i)\end{array}\right\}$ in the output quantum state. Here $k$ and $k^{\prime}$ denote the indices of the coefficients in each such set. Then,

$$
\sum_{k} \alpha_{(h w)^{(i)}}^{k}=\sum_{k^{\prime}} \beta_{(h w)^{(i)}}^{k^{\prime}}
$$

for any $n$-qubit case. This is a powerful result since it, along with the normalization condition, helps us determine the kinds of states that are derivable from the operation of just Power-of-SWAP gates on a general $n$-qubit state.

\section{Conclusions}

In this paper, I have found a way to generate all the basis vector-states that span the space of states accessible by an arbitrary Power-of-SWAP gate. In doing so, we have found several classes of states. These include maximally entangled states such as the W-states, partially entangled cluster states and separable states. Each of these can be used for various kinds of applications in quantum information processing.

With the exponential scaling of the computation basis with the number of basis, we see that the basis vectors of the invariant subspaces also increase exponentially. It was found that only specific states can be generated using an initial quantum state and a combination of $S W A P^{\alpha}$ gates, and hopping between these points represents a quantum computation. By understanding the invariant subspaces, I realize an accessible resource for quantum computation using $S W A P^{a}$. This quantum computation is resilient to parity changes and other forms of errors due to the permutation symmetries of the vectors comprising the invariant subspaces. I hope that this paper will pave the way for the use of the $S W A P^{\alpha}$ for generation of quantum resource-states in quantum information processing tasks.

\section{Acknowledgements}

I would like to acknowledge the contribution of Trinity College, Cambridge and the Nehru Trust for Cambridge University (NTCU) who funded the project. I would like to acknowledge the contributions of Prof. Crispin Barnes, Mr. Yordan Yardanov, Dr. David R. M. Arvidsson-Shukur and Mr. Sathyageeshwar Subramaniam.

\section{Conflicts of Interest}

The author declares no conflicts of interest regarding the publication of this paper.

\section{References}

[1] Einstein, A., Podolsky, B. and Rosen, N. (1935) Can Quantum-Mechanical Description of Physical Reality Be Considered Complete? Physical Review, 47, 777. https://doi.org/10.1103/PhysRev.47.777

[2] Schrodinger, E. (1935) Die gegenwärtige Situation in der Quantenmechanik. Na- 
turwissenschaften, 23, 807-812. https://doi.org/10.1007/BF01491891

[3] Horodecki, R., Horodecki, P., Horodecki, M. and Horodecki, K. (2009) Quantum Entanglement. Reviews of Modern Physics, 81, 865.

https://doi.org/10.1103/RevModPhys.81.865

[4] Amico, L., Fazio, R., Osterloh, A. and Vedral, V. (2008) Entanglement in Many-Body Systems. Reviews of Modern Physics, 80, 517. https://doi.org/10.1103/RevModPhys.80.517

[5] Jurcevic, P., Lanyon, B.P., Hauke, P., Hempel, C., Zoller, P., Blatt, R. and Roos, C.F. (2014) Quasiparticle Engineering and Entanglement Propagation in a Quantum Many-Body System. Nature, 511, 202-205. https://doi.org/10.1038/nature13461

[6] Islam, R., Ma, R., Preiss, P.M., Tai, M.E., Lukin, A., Rispoli, M. and Greiner, M. (2015) Measuring Entanglement Entropy in a Quantum Many-Body System. Nature, 528, 77-83. https://doi.org/10.1038/nature15750

[7] Eggeling, T. and Werner, R. (2001) Separability Properties of Tripartite States with UUU Symmetry. Physical Review A, 63, Article ID: 042111.

https://doi.org/10.1103/PhysRevA.63.042111

[8] Toth, G. and Guhne, O. (2010) Separability Criteria and Entanglement Witnesses for Symmetric Quantum States. Applied Physics B, 98, 617.

[9] Toth, G. and Guhne, O. (2009) Entanglement and Permutational Symmetry. Physical Review Letters, 102, Article ID: 170503.

[10] Markham, D.J. (2011) Entanglement and Symmetry in Permutation-Symmetric States. Physical Review A, 83, Article ID: 042332.

https://doi.org/10.1103/PhysRevA.83.042332

[11] Hubener, R., Kleinmann, M., Wei, T.-C., Gonzalez Guillen, C. and Guhne, O. (2009) Geometric Measure of Entanglement for Symmetric States. Physical Review $A$, 80, Article ID: 032324. https://doi.org/10.1103/PhysRevA.80.032324

[12] Barasinski, A. and Nowotarski, M. (2017) Quantifying Entanglement Properties of Qudit Mixed States with Incomplete Permutation Symmetry. Physical Review A, 95, Article ID: 042333. https://doi.org/10.1103/PhysRevA.95.042333

[13] Calixto, M., Castanos, O. and Romera, E. (2017) Entanglement and Quantum Phase Diagrams of Symmetric Multi-Qubit Systems. Journal of Statistical Mechanics: Theory and Experiment, 2017, Article ID: 103103. https://doi.org/10.1088/1742-5468/aa8703

[14] Kadiri, G. and Sivakumar, S. (2017) Permutation Symmetry and Entanglement in Quantum States of Heterogeneous Systems.

[15] Vogel, K., Akulin, V. and Schleich, W. (1993) Quantum State Engineering of the Radiation Field. Physical Review Letters, 71, 1816-1819. https://doi.org/10.1103/PhysRevLett.71.1816

[16] Makhlin, Y., Schon, G. and Shnirman, A. (2001) Quantum-State Engineering with Josephson-Junction Devices. Reviews of Modern Physics, 73, 357. https://doi.org/10.1103/RevModPhys.73.357

[17] DellAnno, F., De Siena, S. and Illuminati, F. (2006) Multiphoton Quantum Optics and Quantum State Engineering. Physics Reports, 428, 53-168. https://doi.org/10.1016/j.physrep.2006.01.004

[18] Verstraete, F., Wolf, M.M. and Cirac, J.I. (2009) Quantum Computation and Quantum-State Engineering Driven by Dissipation. Nature Physics, 5, 633-636. https://doi.org/10.1038/nphys1342

[19] Chiu, C.S., Ji, G., Mazurenko, A., Greif, D. and Greiner, M. (2018) Quantum State 
Engineering of a Hubbard System with Ultracold Fermions. Physical Review Letters, 120, Article ID: 243201. https://doi.org/10.1103/PhysRevLett.120.243201

[20] Mathonet, P., Krins, S., Godefroid, M., Lamata, L., Solano, E. and Bastin, T. (2010) Entanglement Equivalence of $N$-Qubit Symmetric States. Physical Review A, 81, Article ID: 052315. https://doi.org/10.1103/PhysRevA.81.052315

[21] Ji, Q., Liu, Y., Yin, X., Liu, X. and Zhang, Z. (2013) Quantum Operation Sharing with Symmetric and Asymmetric W States. Quantum Information Processing, 12, 2453-2464. https://doi.org/10.1007/s11128-013-0533-9

[22] Jiang, D.-Y., Wu, R., Li, S. and Wang, Z. (2009) Controlled Dense Coding with Symmetric State. International Journal of Theoretical Physics, 48, 2297-2304. https://doi.org/10.1007/s10773-009-0018-x

[23] Liu, X.-W. (2015) Controlled Dense Coding with Non-Symmetric Quantum State. International Journal of Theoretical Physics, 54, 1253-1257. https://doi.org/10.1007/s10773-014-2322-3

[24] Rajagopal, A. and Rendell, R. (2002) Robust and Fragile Entanglement of Three Qubits: Relation to Permutation Symmetry. Physical Review A, 65, Article ID: 032328. https://doi.org/10.1103/PhysRevA.65.032328

[25] Zanardi, P. (1999) Symmetrizing Evolutions. Physics Letters A, 258, 77-82. https://doi.org/10.1016/S0375-9601(99)00365-5

[26] Eltschka, C. and Siewert, J. (2012) Entanglement of Three-Qubit Greenberger-Horne-Zeilinger-Symmetric States. Physical Review Letters, 108, Article ID: 020502. https://doi.org/10.1103/PhysRevLett.108.020502

[27] Barenco, A., Berthiaume, A., Deutsch, D., Ekert, A., Jozsa, R. and Macchiavello, C. (1997) Stabilization of Quantum Computations by Symmetrization. SIAM Journal on Computing, 26, 1541-1557. https://doi.org/10.1137/S0097539796302452

[28] Wang, X. and Zanardi, P. (2002) Quantum Entanglement and Bell Inequalities in Heisenberg Spin Chains. Physics Letters A, 301, 1-6. https://doi.org/10.1016/S0375-9601(02)00885-X

[29] Wei, J.-H., Qi, B., Dai, H.-Y., Huang, J.-H. and Zhang, M. (2015) Deterministic Generation of Symmetric Multi-Qubit Dicke States: An Application of Quantum Feedback Control. IET Control Theory \& Applications, 9, 2500. https://doi.org/10.1049/iet-cta.2014.1314

[30] Thiel, C., Von Zanthier, J., Bastin, T., Solano, E. and Agarwal, G. (2007) Generation of Symmetric Dicke States of Remote Qubits with Linear Optics. Physical Review Letters, 99, Article ID: 193602. https://doi.org/10.1103/PhysRevLett.99.133603

[31] Shao, X.-Q., Chen, L., Zhang, S., Zhao, Y.-F. and Yeon, K.-H. (2010) Deterministic Generation of Arbitrary Multi-Atom Symmetric Dicke States by a Combination of Quantum Zeno Dynamics and Adiabatic Passage. EPL (Europhysics Letters), 90, Article ID: 50003. https://doi.org/10.1209/0295-5075/90/50003

[32] Nielsen, M. and Chuang, I. (2010) Quantum Computation and Quantum Information. 10th Anniversary Edition, Cambridge University Press, Cambridge. https://doi.org/10.1017/CBO9780511976667

[33] DiVincenzo, D.P., Bacon, D., Kempe, J., Burkard, G. and Whaley, K.B. (2000) Universal Quantum Computation with the Exchange Interaction. Nature, 408, 339-342. https://doi.org/10.1038/35042541

[34] Barnes, C., Shilton, J. and Robinson, A. (2000) Quantum Computation Using Electrons Trapped by Surface Acoustic Waves. Physical Review B, 62, 8410. https://doi.org/10.1103/PhysRevB.62.8410 
[35] Rohling, N. and Burkard, G. (2012) Universal Quantum Computing with Spin and Valley States. New Journal of Physics, 14, Article ID: 083008. https://doi.org/10.1088/1367-2630/14/8/083008

[36] Lin, G.-W., Zou, X.-B., Ye, M.-Y., Lin, X.-M. and Guo, G.-C. (2008) Quantum SWAP Gate in an Optical Cavity with an Atomic Cloud. Physical Review A, 77, Article ID: 064301. https://doi.org/10.1103/PhysRevA.77.064301

[37] Sangouard, N., Lacour, X., Guerin, S. and Jauslin, H.-R. (2005) Fast SWAP Gate by Adiabatic Passage. Physical Review A, 72, Article ID: 062309. https://doi.org/10.1103/PhysRevA.72.062309

[38] Wei, H.-R. and Deng, F.-G. (2015) Compact Implementation of the (SWAP) ${ }^{\alpha}$ Gate on Diamond Nitrogen-Vacancy Centers Coupled to Resonators. Quantum Information Processing, 14, 465-477. https://doi.org/10.1007/s11128-014-0868-x

[39] Lin, X., Yang, R.-C. and Chen, X. (2015) Implementation of a Quantum $\sqrt{\text { swap }}$ Gate for Two Distant Atoms Trapped in Separate Cavities. International Journal of Quantum Information, 13, Article ID: 1550003. https://doi.org/10.1142/S0219749915500033

[40] Liu, A.-P., Cheng, L.-Y., Zhang, S., Zhao, Y., Gao, X.-Z., Chang, Y.-H. and Wang, A.-P. (2016) Deterministic Controlled-Phase Gate and SWAP Gate with Dipole-Induced Transparency in the Weak-Coupling Regime. Optics Communications, 379, 19-24. https://doi.org/10.1016/j.optcom.2016.05.054

[41] Ono, T., Okamoto, R., Tanida, M., Hofmann, H.F. and Takeuchi, S. (2017) Implementation of a Quantum Controlled-SWAP Gate with Photonic Circuits. Scientific Reports, 7, Article No. 45353. https://doi.org/10.1038/srep45353

[42] Borne, A., Bechler, O., Rosenblum, S., Guendelman, G., Mor, O., Netzer, M., Gurovich, D., Ohana, T., Aqua, Z., Drucker, N., Lovsky, Y., Bruch, R., Finkelstein, R., Shafir, E. and Dayan, B. (2017) Demonstration of Deterministic and Passive Photon-Atom SWAP Quantum Gate. In: Quantum Information and Measurement (QIM), Optical Society of America, QT2A-7.

[43] Burkard, G., Loss, D., DiVincenzo, D.P. and Smolin, J.A. (1999) Physical Optimization of Quantum Error Correction Circuits. Physical Review B, 60, Article ID: 11404. https://doi.org/10.1103/PhysRevB.60.11404

[44] Barenco, A., Bennett, C.H., Cleve, R., DiVincenzo, D.P., Margolus, N., Shor, P., Sleator, T., Smolin, J.A. and Weinfurter, H. (1995) Elementary Gates for Quantum Computation. Physical Review A, 52, 3457-3467.

https://doi.org/10.1103/PhysRevA.52.3457

[45] Tanamoto, T., Liu, Y.-X., Hu, X. and Nori, F. (2009) Efficient Quantum Circuits for One-Way Quantum Computing. Physical Review Letters, 102, Article ID: 100501. https://doi.org/10.1103/PhysRevLett.102.100501

[46] Raussendorf, R. and Briegel, H.J. (2001) A One-Way Quantum Computer. Physical Review Letters, 86, 5188-5191. https://doi.org/10.1103/PhysRevLett.86.5188

[47] Fan, H., Roychowdhury, V. and Szkopek, T. (2005) Optimal Two-Qubit Quantum Circuits Using Exchange Interactions. Physical Review A, 72, Article ID: 052323. https://doi.org/10.1103/PhysRevA.72.052323

[48] Guerrero, R.J. and Rojas, F. (2008) Effect of the Dzyaloshinski-Moriya Term in the Quantum (SWAP) ${ }^{\alpha}$ Gate Produced with Exchange Coupling. Physical Review A, 77, Article ID: 012331. https://doi.org/10.1103/PhysRevA.77.012331

[49] Balakrishnan, S. and Sankaranarayanan, R. (2008) Entangling Characterization of SWAP $^{1 / \mathrm{m}}$ and Controlled Unitary Gates. Physical Review A, 78, Article ID: 052305. https://doi.org/10.1103/PhysRevA.78.052305 
[50] Ford, C.J.B. (2017) Transporting and Manipulating Single Electrons in Surface-Acoustic-Wave Minima. Physica Status Solidi B, 254, Article ID: 052305. https://doi.org/10.1002/pssb.201600658

[51] Weyl, H. (2016) The Classical Groups: Their Invariants and Representations. Princeton University Press, Princeton. https://doi.org/10.2307/j.ctv3hh48t

[52] Anshelevich, M., Gaikema, M., Hansalik, M., He, S. and Mehlhop, N. (2017) Expansion of Permutations as Products of Transpositions. arXiv Preprint arXiv: 1702.06093.

[53] Yamanouchi, T. (1937) On the Construction of Unitary Irreducible Representations of the Symmetric Group. Proceedings of the PhysicoMathematical Society of Japan, 3 rd Series, 19, 436.

[54] Gessel, I. and Viennot, G. (1985) Binomial Determinants, Paths, and Hook Length Formulae. Advances in Mathematics, 58, 300-321. https://doi.org/10.1016/0001-8708(85)90121-5

[55] Fulton, W. (1997) Young Tableaux: With Applications to Representation Theory and Geometry. Vol. 35, Cambridge University Press, Cambridge. https://doi.org/10.1017/CBO9780511626241

[56] Kung, J.P. (2014) Young Tableaux in Combinatorics, Invariant Theory, and Algebra: An Anthology of Recent Work. Elsevier, Amsterdam.

[57] Van Beveren, E. (1998) Some Notes on Group Theory. Lecture Notes, Universidade de Coimbra.

[58] Verstraete, F., Dehaene, J., De Moor, B. and Verschelde, H. (2002) Four Qubits Can Be Entangled in Nine Different Ways. Physical Review A, 65, Article ID: 052112. https://doi.org/10.1103/PhysRevA.65.052112

[59] Bengtsson, I. and Zyczkowski, K. (2017) Geometry of Quantum States: An Introduction to Quantum Entanglement. Cambridge University Press, Cambridge. https://doi.org/10.1017/9781139207010

[60] Spee, C., de Vicente, J., Sauerwein, D. and Kraus, B. (2017) Entangled Pure State Transformations via Local Operations Assisted by Finitely Many Rounds of Classical Communication. Physical Review Letters, 118, Article ID: 040503. https://doi.org/10.1103/PhysRevLett.118.040503

[61] Sadhukhan, D., Roy, S.S., Pal, A.K., Rakshit, D., Sen, A., Sen, U., et al. (2017) Multipartite Entanglement Accumulation in Quantum States: Localizable Generalized Geometric Measure. Physical Review A, 95, Article ID: 022301. https://doi.org/10.1103/PhysRevA.95.022301

[62] Devi, A.U., Rajagopal, A., et al. (2012) Majorana Representation of Symmetric Multiqubit States. Quantum Information Processing, 11, 685-710.

https://doi.org/10.1007/s11128-011-0280-8 